\title{
A New Biological Small- and Wide-Angle X-ray Scattering Beamline Featured in High Flux, USAXS, and Microbeam at Taiwan Photon Source
}

\section{Kuei-Fen Liao ${ }^{1}$, Yi-Qi Yeh, ${ }^{1}$ Orion Shih, ${ }^{1}$ Chun-Jen Su, ${ }^{1}$ Wei-Ru Wu, ${ }^{1}$ Chen-An Wang, ${ }^{1}$ Din-Goa Liu, ${ }^{1}$ Chien-Hung Chang, ${ }^{1}$ Liang-Chih Chiang, ${ }^{1}$ Cheng-Yuan Lin, ${ }^{1}$ Chia-Feng Chang, ${ }^{1}$ Cheng-Chih Liang, ${ }^{1}$ Te-Hui Lee, ${ }^{1}$ Meng-Chiao Ho, ${ }^{2}$ and U-Ser Jeng ${ }^{1,3^{*}}$}

${ }^{I}$ National Synchrotron Radiation Research Center, Hsinchu Science Park, Hsinchu 30076, Taiwan;

\author{
${ }^{2}$ Institute of biochemical sciences \& Institute of biological chemistry, Academia Sinica, Nankang, Taipei 115, Taiwan,
}

${ }^{3}$ Department of Chemical Engineering, National Tsing Hua University, Hsinchu 30013,

usjeng@nsrrc.org.tw

\begin{abstract}
A new biological small-angle X-ray scattering (BioSAXS) beamline is developed with the 3.0 GeV Taiwan Photon Source (TPS), for studies of biological structures in a wide range of length and time scales. The beamline provides a high flux $\left(4 \times 10^{14}\right.$ photons $\left./ \mathrm{s}\right)$ for time-resolved and synchronized small- and wide-angle X-ray scattering (SAXS-WAXS), and offers new opportunities for ultra-SAXS (USAXS) to resolve the hierarchical structures of bio-machinery assemblies in solution, gel or condensed forms and anomalous SAXS/WAXS for metal or mineral distributions and compositions in an organelle or drug carrier. The beamline application extends to microbeam SAXS/WAXS for correlated crystal and nanostructural mappings in natural fibril tissues and synthetic biomaterials under tailored environmental controls. Concomitant SAXS-WAXS data collections are realized with a unique detecting system comprising an Eiger X-9M detector for SAXS and a custom-designed Eiger X-1M detector for simultaneous WAXS. These two X-ray detectors (75 $\mu \mathrm{m}$ pixel resolution) move independently with multi-degrees of freedom inside a large vacuum vessel of $12 \mathrm{~m}$ long and $1.5 \mathrm{~m}$ dia., providing dynamic and fast changes in detecting configuration for optimized data collections. Solution SAXS and WAXS of biomacromolecules are facilitated with an integrated system of online sample purification system of HPLC, strengthened by onsite UV-vis absorption followed by refractive of index measurement in one sample elution. The beamline has been opened to users since September 2020.
\end{abstract}

Keywords: BioSAXS, USAXS, WAXS 